\title{
Note on weakly inward mappings
}

\author{
by MiChal FEČKAN (Bratislava)
}

Abstract. The Nielsen fixed point theory is used to show several results for certain operator equations involving weakly inward mappings.

1. Introduction. In this note, we study the equation

$$
u=\varepsilon F(u, \lambda), \quad 0=B(u, \lambda),
$$

where $F: X \times \mathbb{R}^{m} \rightarrow X$ and $B: X \times \mathbb{R}^{m} \rightarrow \mathbb{R}^{m}$ are continuous, and $X$ is a Banach space. Furthermore, $F$ is compact and $B$ is globally Lipschitz in $u$ with a constant $M$. We are interested in the lower bound for the number of parameters $\lambda$ for which (1.1) has a solution $u \in D$ for any small $\varepsilon>0$. Throughout this paper $D$ is a bounded, closed, convex, nonempty subset of $X$.

For this purpose, we shall employ the Nielsen fixed point theory [6] as in $[1,4]$. Our method is similar to that of the last two papers. That method can be applied only for specific nonlinearities $F$. The aim of the paper is to show that the results of $[1,4]$ can be extended to the case when $F(\cdot, \lambda)$ is weakly inward with respect to $D$ for any $\lambda \in \mathbb{R}^{m}$.

We were motivated by [5] for the study of the problem (1.1), and we show that nonlinearities suggested by [5] will be sufficient for our purpose.

2. Preliminaries. Throughout this paper, $X^{*}$ denotes the dual space of $X$. For any $x \in D$, we define the weakly inward set of $D$ at $x$ to be $\bar{I}_{D}(x)$, where

$$
I_{D}(x)=\{x+t(y-x) \mid t \geq 0, y \in D\} .
$$

A mapping $T: D \rightarrow X$ is said to be weakly inward with respect to $D$ if $T(x) \in \bar{I}_{D}(x)$ for all $x \in D$. By [3, Lemma 18.2] we have

1991 Mathematics Subject Classification: 47H15, 47H17.

Key words and phrases: weakly inward maps, Nielsen fixed point theory, retractability. 
Proposition 2.1. T:D $\rightarrow X$ is weakly inward with respect to $D$ if and only if

$x \in \partial D, x^{*} \in X^{*}$, and $x^{*}(x)=\sup _{D} x^{*}(y)$ imply $x^{*}(T(x)) \leq x^{*}(x)$.

Now we recall some definitions from $[1,4,5]$.

Definition 2.2. Suppose that $r: W \rightarrow A$ is a map, $W, A$ are subsets of $X$ and $A \subset W$. If $r(a)=a$ for each $a \in A$ then $r$ is called a retraction of $W$ to $A$ and $A$ is called a retract of $W$.

Definition 2.3. Suppose that $T: X \rightarrow X$ is a map and $W$ is a subset that retracts onto a subset $Q$ of itself by a retraction $r: W \rightarrow Q$. We shall say that $T$ is $\mu$-retractable onto $Q$ with a retraction $r$ if

$\{x \in X \mid$ there exists $a \in T(Q)$ such that $|x-a|<\mu\} \subset W$

and

$$
\text { if } y \in W \backslash Q \text { and } r(y)=x \text { then }|y-T(x)|>\mu \text {. }
$$

We shall say that $T$ is retractable onto $Q$ with a retraction $r$ if $T(Q) \subset W$ and if $y \in W \backslash Q$ and $r(y)=x$ implies $y \neq T(x)$.

We see that if $T$ is $\mu$-retractable onto $Q$ then any perturbation $\mathcal{T}: X \rightarrow$ $X$ of $T$ with error $\mu$ on $Q$, i.e. $|\mathcal{T}(x)-T(x)|<\mu$ for $x \in Q$, is still retractable onto $Q$. The main advantage of this definition 2.3 is the following: If $T$ is retractable onto $Q$ with a retraction $r: W \rightarrow Q$ then the map $r \circ T: Q \rightarrow Q$ has a fixed point $x \in Q$ if and only if $T(x)=x$.

Definition 2.4. $D \subset X$ is said to be a retract of $X$ with the property (P) if there is a retraction $r: X \rightarrow D$ such that for any $x \in X \backslash D$ there is an $x^{*} \in X^{*}$ such that

$$
x^{*}(x)>x^{*}(r(x))=\sup _{D} x^{*}(y)
$$

By [5] we have

Proposition 2.5. $D$ is a retract of $X$ with the property $(\mathrm{P})$ if one of the following conditions is satisfied:

(a) $D^{0}$, the interior of $D$, is not empty;

(b) $X$ is a reflexive Banach space;

(c) There is a metric projection from $X$ onto $D$.

3. Main results. We are ready to state the main results of the paper.

Proposition 3.1. Assume $D$ is a retract of $X$ with the property $(\mathrm{P})$; let $r$ be the corresponding retraction. If $T: D \rightarrow X$ is weakly inward with respect to $D$, then $T$ is retractable onto $D$ with retraction $r$. 
Proof. Assume there is $z=T(x)$ such that $r(z)=x$ and $z \notin D$. Then $r(z)=x \in \partial D$. Hence $x^{*} \in X^{*}$ and $x^{*}(x)=\sup _{D} x^{*}(y)$ imply $x^{*}(T(x)) \leq x^{*}(x)$. This implies

$$
x^{*}(r(z))=x^{*}(x)=\sup _{D} x^{*}(y) \geq x^{*}(z),
$$

which is a contradiction to $(\mathrm{P})$ in Definition 2.4. The proof is finished.

Remark 3.2. Consider $B=D=\{x \in X|| x \mid \leq 1\}$ for a Hilbert space $X$. Then by Proposition 2.5(c), $B$ is a retraction of $X$ with the property $(\mathrm{P})$ by means of the retraction

$$
r(x)= \begin{cases}x /|x|, & |x| \geq 1 \\ x, & |x| \leq 1\end{cases}
$$

On the other hand, we see that $T: B \rightarrow X$ is retractable onto $B$ with retraction $r$ if and only if

$$
x \neq \gamma T(x) \quad \forall 0<\gamma<1, \forall x,|x|=1 .
$$

Of course, such a mapping can be not weakly inward with respect to $D$. Thus, the retractability in the framework of Theorem 3.1 is, generally, more than the weak inwardness.

We refer the reader to the book [6] for the definition of the Nielsen number of mappings and its basic properties.

THeOREm 3.3. Suppose that there are a constant $\mu>0$ and a compact, locally contractible subset $S$ of $\mathbb{R}^{m}$ such that the map

$$
\Pi(\lambda)=\lambda+B(0, \lambda)
$$

is $\mu$-retractable onto $S$ with a retraction $\pi$. Furthermore, assume $F(\cdot, \lambda)$, for $\lambda \in S$, is weakly inward with respect to $D$ for $D$ being a retraction of $X$ with the property $(\mathrm{P})$ and $0 \in D$. Then for any small $\varepsilon>0$, there are at least $N(\pi \circ \Pi)$ (the Nielsen number of the map $\pi \circ \Pi: S \rightarrow S$ ) parameters $\lambda \in S$ for which the equation (1.1) has a solution in $D$.

Proof. We follow $[1,4]$ by transforming (1.1) into the following fixed point problem:

$$
T_{\varepsilon}(u, \lambda)=(u, \lambda),
$$

where

$$
T_{\varepsilon}: D \times S \rightarrow X \times \mathbb{R}^{m}, \quad T_{\varepsilon}(u, \lambda)=(\varepsilon F(u, \lambda), \lambda+B(\varepsilon F(u, \lambda), \lambda)) .
$$

Since $0 \in D$, we see that $\varepsilon F(\cdot, \lambda)$ is weakly inward with respect to $D$ for any $\lambda \in S$ and $0<\varepsilon \leq 1$. By Proposition 3.1 and [1, 4], we can easily see that $T_{\varepsilon}$ is retractable onto $D \times S$ with retraction

$$
r \times \pi: X \times W \rightarrow D \times S,
$$


for $\varepsilon>0$ sufficiently small, where $r$ is the retraction from Definition 2.4 and $W$ is a subset of $\mathbb{R}^{m}$ with $S \subset W$ from Definition 2.3. Indeed, assume

$$
(r \times \pi) \circ T_{\varepsilon}\left(u_{0}, \lambda_{0}\right)=\left(u_{0}, \lambda_{0}\right)
$$

for some $\left(u_{0}, \lambda_{0}\right) \in D \times S$. Then

$$
r \circ\left(\varepsilon F\left(u_{0}, \lambda_{0}\right)\right)=u_{0}, \quad \pi \circ\left(\lambda_{0}+B\left(\varepsilon F\left(u_{0}, \lambda_{0}\right), \lambda_{0}\right)\right)=\lambda_{0} .
$$

By Proposition 3.1, $u_{0}=\varepsilon F\left(u_{0}, \lambda_{0}\right)$ for $1 \geq \varepsilon>0$. Furthermore, for $\varepsilon>0$ small the mapping $\lambda+B(\varepsilon F(u, \lambda), \lambda)$ is a perturbation on $D \times S$ of $\Pi(\lambda)=\lambda+B(0, \lambda)$ with error $\mu$. By the $\mu$-retractability of $\Pi, \lambda_{0}+$ $B\left(\varepsilon F\left(u_{0}, \lambda_{0}\right), \lambda_{0}\right)=\lambda_{0}$ and retractability of $T_{\varepsilon}$ onto $D \times S$ with retraction $r \times \pi$ is proved.

Hence $T_{\varepsilon}$ has at least $N\left((r \times \pi) \circ T_{\varepsilon}\right)$ fixed points in $D \times S$. Note that we can indeed use the Nielsen fixed point theory, since $D \times S$ is an absolute neighbourhood retract and $T_{\varepsilon}$ is a compact, continuous mapping.

On the other hand,

$$
N\left((r \times \pi) \circ T_{\varepsilon}\right)=N\left((r \times \pi) \circ T_{0}\right)=N(\pi \circ \Pi) .
$$

Thus $T_{\varepsilon}$ has at least $N(\pi \circ \Pi)$ fixed points in $D \times S$ for any $\varepsilon>0$ small. The proof is finished.

Remark 3.4. As pointed out in $[1,4]$, the smallness of $\varepsilon>0$ depends on the $\mu$-retractability of $S$. The larger $\mu$, the bigger $\varepsilon$. More precisely, the assertion of Theorem 3.3 holds for any $1 \geq \varepsilon>0$ such that

$$
\mu>\varepsilon M \sup _{D \times S}|F(u, \lambda)| \text {. }
$$

Indeed, we know that both $\Pi(\lambda)=\lambda+B(0, \lambda)$ is $\mu$-retractable onto $S$ with retraction $\pi$, and

$$
|B(\varepsilon F(u, \lambda), \lambda)-B(0, \lambda)| \leq M \varepsilon|F(u, \lambda)|<\mu
$$

for any $(u, \lambda) \in D \times S$. Now applying the arguments preceding Definition 2.4 as in the proof of Theorem 3.3, we see the sufficiency of this inequality.

To be more concrete, we consider the case $m=2$. Moreover, let $S=\mathcal{A}=$ $\left\{\lambda \in \mathbb{R}^{2}|1 / 2 \leq| \lambda \mid \leq 1\right\}$ be the circular ring (annulus) with the retraction $[2],[4]$

$$
\varrho(\lambda)= \begin{cases}\lambda /(2|\lambda|), & 0<|\lambda|<1 / 2, \\ \lambda, & 1 / 2 \leq|\lambda| \leq 2, \\ 2 \lambda /|\lambda|, & 2<|\lambda| .\end{cases}
$$

Then we can construct a map $g$ according to $[2$, p. 54] such that $g$ is $\mu$ retractable onto $\mathcal{A}$ with retraction $\varrho$ for a $\mu>0$ small. For instance, we can take $g(\lambda)=q(|\lambda|) \lambda^{k}$ (we identify $\mathbb{R}^{2}$ with $\mathbb{C}$, the complex plane) satisfying

$$
q(1 / 2) / 2^{k} \geq 1 / 2+\mu, \quad q(b) b^{k}>\mu \quad \text { for } 1 / 2 \leq b \leq 1,
$$




$$
2^{k} q(2) \leq 2-\mu, \quad k \in \mathbb{N} \backslash\{1\} .
$$

We see that the above conditions for $q$ are precisely the assumptions of [2, Proposition 1.5] for the map $g(\lambda)=q(|\lambda|) \lambda^{k}$. Finally, we know [2] that $N(\varrho \circ g)=\left|\operatorname{deg} \lambda^{k}-1\right|=k-1$.

By applying Theorem 3.3, we obtain

TheOREM 3.5. Consider (1.1) with $m=2$. Furthermore, assume $F(\cdot, \lambda)$, for $\lambda \in \mathcal{A}$, is weakly inward with respect to $D$ for $D$ being a retract of $X$ with the property $(\mathrm{P})$ and $0 \in D$. Suppose $B(0, \lambda)=g(\lambda)-\lambda$ for $\lambda \in \mathcal{A}$ and for $g$ defined above. Then for any $1 \geq \varepsilon>0$ satisfying

$$
\mu>\varepsilon M \sup _{D \times \mathcal{A}}|F(u, \lambda)|,
$$

there are at least $k-1$ parameters $\lambda \in \mathcal{A}$ for which the equation (1.1) has a solution in $D$.

\section{References}

[1] R. F. Brown, Nielsen fixed point theory and parametrized differential equations, in: Contemp. Math. 72, Amer. Math. Soc., 1989, 33-46.

[2] - Topological identification of multiple solutions to parametrized nonlinear equations, Pacific J. Math. 131 (1988), 51-69.

[3] K. Deimling, Nonlinear Functional Analysis, Springer, New York, 1985.

[4] M. Fečkan, Nielsen fixed point theory and nonlinear equations, J. Differential Equations 106 (1993), 312-331.

[5] S. Hu and Y. Sun, Fixed point index for weakly inward mappings, J. Math. Anal. Appl. 172 (1993), 266-273.

[6] B. Jiang, Lectures on Nielsen Fixed Point Theory, Contemp. Math. 14, Amer. Math. Soc., 1983.

DEPARTMENT OF MATHEMATICAL ANALYSIS

FACULTY OF MATHEMATICS AND PHYSICS, COMENIUS UNIVERSITY

MLYNSKÁ DOLINA, 84215

BRATISLAVA, SLOVAKIA

Reçu par la Rédaction le 6.5.1993

Révisé le 20.2.1995 